\title{
On the effect of contraction ratio in viscoelastic flow through abrupt contractions
}

\author{
Manuel A. Alves ${ }^{\mathrm{a}}$, Paulo J. Oliveira ${ }^{\mathrm{b}, *}$, Fernando T. Pinho $^{\mathrm{c}}$ \\ ${ }^{a}$ Departamento de Engenharia Química, CEFT, Faculdade de Engenharia da Universidade do Porto, \\ Rua Dr. Roberto Frias, 4200-465 Porto, Portugal \\ ${ }^{\mathrm{b}}$ Departamento de Engenharia Electromecânica Unidade de Materiais Têxteise Papeleiros, \\ Universidade da Beira Interior, 6201-001Covilhã, Portugal \\ ${ }^{\mathrm{c}}$ Centro de Estudos de Fenómenos de Transporte, Depart. Eng. Mecânica, Universidade do Minho, \\ Campus de Azurém, 4800-058 Guimarães, Portugal
}

Received 10 July 2003; received in revised form 24 October 2003; accepted 5 January 2004

This article is part of a Special Volume containing papers from the XIIIth International Workshop on Numerical Methods in Viscoelastic Flows

\begin{abstract}
A numerical study of the creeping flow of a PTT fluid through planar sudden contractions was carried out to quantify the effect of contraction ratio upon the flow characteristics (streamlines and size and intensity of recirculation vortices). The relevant governing equations were solved with a finite volume method embodying a new high-resolution scheme (Alves et al. [Int. J. Numer. Meth. Fluids 41 (2003) 47]) for the discretisation of convection terms, which is here explained and shown to yield improved accuracy and robustness. The results of the simulations, in terms of streamline patterns, give further evidence for a lip-vortex enhancement mechanism and are in remarkable agreement with flow visualization photographs from the literature. In addition, the results show that the variation of flow features in the vicinity of the re-entrant corner, such as lip vortex size and streamlines, are dominated by downstream quantities and scale with the common definition for the Deborah number in this flow, while flow characteristics in the salient corner region scale with that Deborah number divided by the contraction ratio. (C) 2004 Elsevier B.V. All rights reserved.
\end{abstract}

Keywords: Planar contraction; Viscoelastic flow; Contraction ratio; PTT model

\section{Introduction}

Numerical studies of benchmark flow problems are still of value, not only because they may provide more accurate results to be used in code evaluation, but also because they may highlight some new features in flows which have been simulated only over a limited range of parameters. The planar contraction geometry was put forth as a benchmark problem in 1987, during the fifth international workshop on numerical methods in non-Newtonian flows [2], for the specific contraction ratio of $4: 1$ (relation of upstream and downstream channel heights - see Fig. 1 for flow configuration and main notation). Since then, it has been recognised that the speci-

\footnotetext{
* Corresponding author. Fax: +351 275329972.

E-mail addresses: mmalves@fe.up.pt (M.A. Alves), pjpo@ubi.pt (P.J. Oliveira), fpinho@fe.up.pt (F.T. Pinho).
}

fied ratio of 4:1 was not the best choice as many interesting features of an actual contraction flow are not emphasised, as noted by Nigen and Walters [3]. These authors suggest that flows in contractions having ratios other than the standard 4:1 value should be explored in more detail, but a survey of the literature reveals only a few studies, mainly experimental, where the variation of contraction ratio is considered; see for example works [3-6] which are briefly reviewed in Section 2. Hence, while the existing numerical data for the 4:1 contraction may remain as a valid benchmark data set for assessment of newly developed simulation codes, there is a need for a systematic investigation of the role of contraction ratio on viscoelastic flow through contractions.

This task may be carried out more effectively by numerical simulations and in the current study we investigate the effect of contraction ratio (CR) upon the main overall features of this type of flow. In particular, we look at the variation with 


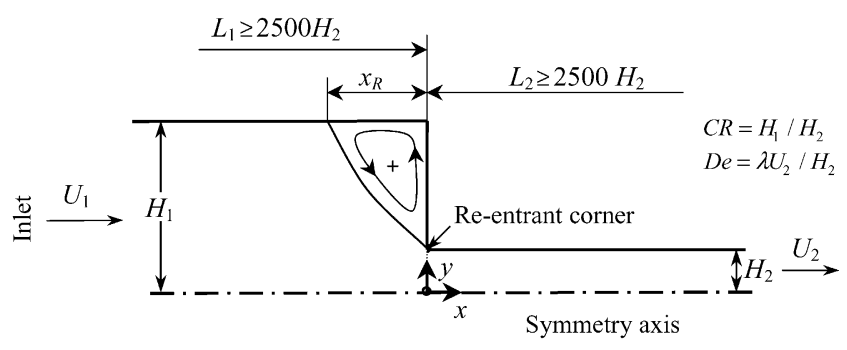

Fig. 1. Schematic of the flow configuration.

Deborah number and contraction ratio of: size and intensity of corner vortex; existence of lip vortex; intensity of lip vortex; and streamline patterns. Thus, this work complements our previous results for the $4: 1$ contraction [7] by adding new results for the following contraction ratios: 10, 20, 40 and 100.

It turns out that many of the complex flow patterns photographed by Evans and Walters [6] are predicted by the simulations and, in addition, some interesting scaling rules regarding unique variations of the main flow features with elasticity are found. Specifically, for the Phan-Thien/Tanner model (PTT, [8]) considered in the present work, and for contraction ratios above about 10 , the corner vortex size $\left(x_{\mathrm{R}}\right)$ and intensity $\left(\Psi_{\mathrm{R}}\right)$ scale with the usual definition for a Deborah number divided by the contraction ratio (De/CR), whereas the lip vortex intensity scales with the Deborah number alone. Such scaling provides a perfect match (universality) of local quantities and this is well illustrated by the collapse of streamline plots for different contraction ratios, when distances are scaled with upstream channel height and the standard Deborah number is modified with CR.

An important aspect in viscoelastic flow computations is the accuracy of the schemes used to represent advective transport in the constitutive equations. A new high-resolution scheme has been developed by the authors [1], aimed at retaining both robustness and higher accuracy than the firstorder, highly diffusive, upwind scheme. A short description of the scheme is here provided and its behaviour is demonstrated.

\section{Review of related work}

This review is mainly concerned with works on sudden contraction flows where the interest was, at least in part, the evaluation of the effect of contraction ratio. For this purpose, the distinction of axisymmetric and planar configurations does not appear as the relevant factor, and so both are considered.

Most works are experimental. Nguyen and Boger [4] examined Boger fluids (dilute solutions of polyacrylamide in glucose syrup) in axisymmetric contractions with $\mathrm{CR}=4.09$, $7.67,12.3$ and 14.83 , with the expressed intention of studying the effect of the contraction ratio. The vortex size results were shown either as $x_{\mathrm{R}} / D_{1}$ versus $W e$, or as the non-dimensional group $(\mathrm{CR} \times W e) /\left(x_{\mathrm{R}} / D_{1}\right)$ versus $E l \equiv \lambda \eta_{0} / \rho D_{2}^{2}$ (the elasticity number). The Weissenberg number was defined as $W e=\lambda U_{2} / D_{2}$, where $D_{1}$ and $D_{2}$ are diameters (corresponding to the $2 \mathrm{H}_{1}$ and $2 \mathrm{H}_{2}$ of our planar configuration; cf. Fig. 1), and the relaxation time $\lambda$ was determined from the measured shear data. In neither case are the data well correlated by those choices of non-dimensionalization.

In a subsequent study, Boger et al. [9] used two constantviscosity fluids (polyacrylamide dissolved in corn syrup and polyisobutylene dissolved in polybutene) flowing through circular contractions with contraction ratios of 4 and 16 . They stated that for the Newtonian fluids (the solvents) the curves of $x_{\mathrm{R}} / D_{1}$ versus $R e$ were independent of $\mathrm{CR}$ and, under creeping flow conditions, also of $R e$. This was corroborated by the experimental data shown. A number of experimental data for viscoelastic fluid flow (from Boger and co-workers) were represented as $x_{\mathrm{R}} / D_{1}$ versus Weissenberg number, $W e \equiv \lambda \dot{\gamma}_{w}=8 \lambda U_{2} / D_{2}$. The authors observed a definite effect of contraction ratio and commented that their previous conclusion in Ref. [4], which considered $\mathrm{CR}$ not to be an important variable when $\mathrm{CR} \geq 4$, was premature.

At about the same time (1986) the two well-known papers of Evans and Walters [6,10] appeared. The first one dealt with planar and square/square contractions, considering both Boger fluids (polyacrylamide dissolved in maltose syrup and water) and shear-thinning fluids (polyacrylamide in water), and three contraction ratios were investigated: 4, 16 and 80. It was shown, for the first time, that for large contraction ratios a lip vortex is formed and becomes the dominant mechanism for vortex enhancement. However, for the case $\mathrm{CR}=4$, such a lip vortex was not present. In essence, the important series of photographs for the shear-thinning fluid in the 4, 16 and 80 contraction ratios are in close (qualitative and semiquantitative) agreement with the current simulations with a PTT fluid (see Section 6.1). In the second paper [10], a lip vortex was generated even in the 4:1 contraction ratio when the concentration of polyacrylamide was lowered $(0.2 \%$ in water). In both studies the question of how the vortex size $\left(x_{\mathrm{R}}\right)$ should be represented as a function of Deborah number, for the various contraction ratios, was not considered.

Further results of Boger fluids through axisymmetric contractions were given by McKinley et al. [11] in an extensive and very interesting work, with emphasis on non-linear transitions of flow patterns for increasing flow rates. The fluid was a semi-dilute solution of $0.31 \%$ polyisobutylene dissolved in a mixture of tethradecane and polybutene, and various contraction ratios were employed $(3,4,5,6$ and 8$)$. With relevance to the present study, the data for vortex size represented as $x_{\mathrm{R}} / D_{2}$ versus Deborah number were shown to vary with contraction ratio when a corner vortex alone was present, and to be approximately invariant with $\mathrm{CR}$ after a lip vortex was formed.

Other authors have carried out experimental work in contraction flows for different contraction ratios. For example, Maia and Binding [12] used the ratios 24.4, 47.9, 67.1, 100 
and 124.3, in an axisymmetric orifice geometry, with the fluid S1 (polyisobutylene dissolved in polybutene). The interest was in obtaining the extensional properties of that fluid, but they also showed that the vortex size varied approximately as $x_{\mathrm{R}} / D_{1} \sim \mathrm{CR}^{-0.4}$. More recently, Nigen and Walters [3] made experiments with Boger fluids (polyacrylamide dissolved in a mixture of glucose syrup and water) in a range of contraction ratios for planar $(\mathrm{CR}=8,16,32$; or 10,20 , 40 ; or 4,8$)$ and round geometries $(\mathrm{CR}=4,8,16,32$; and $2,4,8)$. The main quantitative results were flow rates versus pressure drop curves. For one of the fluids, they showed the variation of vortex sizes as $x_{\mathrm{R}} / D_{1}$ versus $U_{1}$ (dimensional average velocity) for two contraction ratios ( $\mathrm{CR}=8$ and 16 , in the axisymmetric case). Their Fig. 12 showed that with such scaling the vortex size does not collapse onto a single curve.

Most published numerical work is related to the standard case $\mathrm{CR}=4$ (see $[7,13]$ and references therein), and while some authors have used different values, say $\mathrm{CR}=8$ (e.g. [14]), or even both those values [15], we could not find any study with systematic variation of contraction ratio. It appears therefore that a numerical study aimed at assessing the influence of contraction ratio on the main vortex features in a planar contraction, for a typical shear-thinning fluid model (here we shall use the Phan-Thien/Tanner equation), is worth pursuing.

\section{Equations and solution method}

The problem of the flow of a viscoelastic incompressible liquid through a contraction entails the solution of the mass conservation equation,

$\nabla \cdot \boldsymbol{u}=0$

the equation of motion,

$\rho\left[\frac{\partial \boldsymbol{u}}{\partial t}+\nabla \cdot(\boldsymbol{u} \boldsymbol{u})\right]=-\nabla p+\eta_{\mathrm{s}} \nabla \cdot\left(\nabla \boldsymbol{u}+\nabla \boldsymbol{u}^{\mathrm{T}}\right)+\nabla \cdot \boldsymbol{\tau}$,

and a constitutive equation giving the extra stress tensor $\boldsymbol{\tau}$ as a function of the flow kinematics. There are numerous models for this purpose, which may be expressed either as partial differential equations or as integral equations, and the choice is usually made on the basis of better fitting the measured rheometrical properties of an actual fluid. Since the current objective is to extend the simulations of Alves et al. [7], who employed a finite volume method for differential constitutive equations, to cover a range of contraction ratios in contraction flows, it appears natural to follow the same form of the Phan-Thien/Tanner model. This model was elaborated from network theory and it is appropriate for both polymer solutions (most of the fluids used in the experimental work cited in Section 2) and polymer melts; it has also been used in many numerical works dealing with contraction flows (see e.g. [16] and references in [7]), and has been compared with other models and shown to give a better fit of measured properties [17]. From these considerations, in the present work we shall employ the simplified form of the Phan-Thien/Tanner model, defined by the equation:

$$
\begin{aligned}
\lambda\left[\frac{\partial \boldsymbol{\tau}}{\partial t}+\nabla \cdot(\boldsymbol{u} \boldsymbol{\tau})\right]+f(\boldsymbol{\tau}) \boldsymbol{\tau}= & \eta_{\mathrm{p}}\left(\nabla \boldsymbol{u}+\nabla \boldsymbol{u}^{\mathrm{T}}\right) \\
& +\lambda\left(\boldsymbol{\tau} \cdot \nabla \boldsymbol{u}+\nabla \boldsymbol{u}^{\mathrm{T}} \cdot \boldsymbol{\tau}\right),
\end{aligned}
$$

where the simplifications used are a zero contribution from the lower-convected part of the full Gordon-Schowalter derivative and a linear form for the function $f(\boldsymbol{\tau})$ multiplying the stress in the left-hand side of Eq. (3). Hence, we have (from [8]):

$f(\boldsymbol{\tau})=1+\frac{\lambda \varepsilon}{\eta_{\mathrm{p}}} \operatorname{Tr}(\boldsymbol{\tau})$,

where $\lambda$ is the constant relaxation time of the model, $\varepsilon$ an extensional-related parameter of the model and $\eta_{\mathrm{p}}$ a polymerrelated viscosity coefficient, as opposed to the solvent-related $\eta_{\mathrm{s}}$ in Eq. (2). The sum of the two viscosities is $\eta_{0}=\eta_{\mathrm{p}}+\eta_{\mathrm{s}}$, representing the zero-shear-rate viscosity of the model fluid in simple shear flow. In the PTT model the polymer viscosity is shear thinning; the parameter $\eta_{\mathrm{p}}$ representing its zero-shearrate value. A solvent viscosity ratio $\beta=\eta_{\mathrm{s}} / \eta_{0}$ is used to prescribe the amount of $\eta_{\mathrm{s}}$ in comparison to $\eta_{\mathrm{p}}$. In Eq. (4), Tr represents the trace operator.

The above governing equations are solved with the finite volume method of Oliveira et al. [18]. Some of the main characteristics of this method are: (i) a fully collocated variable arrangement, thereby greatly simplifying application of the method to more general non-orthogonal and multiplyconnected flow domains (in fact, the code is already written for such general type of applications, including 3D); (ii) a special coupling algorithm for the three dependent-variable fields $\boldsymbol{u}-p-\boldsymbol{\tau}$ (velocity/pressure/stress); (iii) a truly secondorder scheme for all spatial derivatives in the equations (both diffusion and convection). All these issues were addressed in previous works $[18,13,1,7]$ and shall not be reproduced here, except the important question of the discretisation of the convective terms in the stress equation (3) which will be described in greater detail in Section 4. Otherwise, only a brief outline of the solution procedure is given hereafter.

In broad terms, the above equations are discretised on a computational mesh, are linearised and transformed into algebraic equations which relate the values of each dependent variable at a given cell centre with values in the nearby surrounding cell centres. The time-dependent terms in Eqs. (2) and (3) are retained in the discretisation so that a steadystate solution is effectively approached by a succession of time advancement steps. At each time step, fully-implicit sets of algebraic equations, with a regular pattern of sparsity (five non-zero diagonals for a 2D problem, as in the present case), need to be solved for each dependent variable; this is done with preconditioned conjugate gradient methods. The first equations to be solved are those for the stress tensor 
components (from Eq. (3)), which are solved sequentially for each component by assuming given kinematics (velocity field from the previous time step). Then, the momentum equations (from Eq. (2)) are solved sequentially for each velocity component $(u, v, w)$, with an assumed pressure (from previous time step) and the newly calculated stress field. Finally, the discretised continuity equation (from Eq. (1)) is rearranged into a Poisson pressure-correction equation which is implicitly solved for the pressure correction $p^{\prime}$; this is then added to the pressure from the previous time step and is also used to calculate velocity corrections which provide a new divergence-free velocity field. These steps are repeated until the norm of the residuals of all equations becomes smaller than the convergence tolerance of $10^{-4}$. It has been checked from numerical experiments that this tolerance, in terms of normalised variables, provides an adequately converged solution, which remains virtually unchanged when the tolerance value is lowered (say, to $10^{-6}$ ).

\section{Discretisation of the convection fluxes}

Upon integration over a control-volume, the convective terms in Eqs. (2) and (3) (i.e. terms like $\partial(\rho \boldsymbol{u} \phi) / \partial x$ where $\phi$ is any dependent variable, $u_{i}, \tau_{i j}$, etc.) are written as the net sum of flux contributions on cell faces:

$$
\int_{V} \nabla \cdot(\rho \boldsymbol{u} \phi) \mathrm{d} v=\int_{S} \boldsymbol{n} \cdot(\rho \boldsymbol{u} \phi) \mathrm{d} s \rightarrow \sum_{\mathrm{f}=1}^{6} F_{\mathrm{f}} \phi_{\mathrm{f}},
$$

where $F_{\mathrm{f}}$ are outgoing mass-fluxes across faces and $\mathrm{f}$ denotes the face in question. Generally, in 3D applications, for a hexahedral control volume (with volume $V$ and surface area $S$ ), there are six faces; these reduce to four faces when the application is only 2D. In the constitutive equation the differential fluxes are not multiplied by density (in fact, they may be multiplied by the relaxation time, cf. Eq. (3)) and in this case $F$ represents the volumetric flux. The purpose of the convection scheme is then to specify the values of $\phi_{\mathrm{f}}$ at the face, based on existing values at the neighbouring cell centres.

The simplest scheme satisfying the transportive property is upwind, whereby $\phi_{\mathrm{f}}=\phi_{\mathrm{P}}$ where $\mathrm{P}$ is the cell centre situated on the upwind side in relation to face $\mathrm{f}$ (measured by $F_{\mathrm{f}}>0$ ). Upwind differencing is a first-order scheme. Besides, for the typical differential constitutive equations found in viscoelastic flows, which do not have a diffusion-like term, upwind is too inaccurate because it introduces excessive numerical diffusion errors in the solution. If some physical diffusion were present, then application of upwinding together with a very refined mesh could be acceptable, provided the numerical diffusion coefficient be much smaller than the true diffusion. This problem is accentuated when the constitutive model does not have a "solvent viscosity" contribution, as in the case of the UCM model, and when very sharp stress gradients are present oblique to the mesh directions. Higher-order discretisation schemes are problematic because the bounded- ness property of the original differential operator is difficult to retain, but high-resolution methods have been devised to circumvent such problems; see e.g. Leonard [19], where the philosophy here followed is explained in detail.

The rationale behind these schemes becomes very simple when explained under the normalised variable diagram (NVD) shown in Fig. 2, where the normalised face value of any variable, $\tilde{\phi}_{\mathrm{f}}$, is plotted versus the corresponding centre of cell value:

$\tilde{\phi}_{\mathrm{f}}=f n\left(\tilde{\phi}_{\mathrm{P}}\right) \quad$ with $\quad \tilde{\phi}=\frac{\phi-\phi_{\mathrm{U}}}{\phi_{\mathrm{D}}-\phi_{\mathrm{U}}}$.

The normalisation is such that $\tilde{\phi}_{\mathrm{f}}$ remains between 0 and 1 (bounds imposed by surrounding nodal values), unless the local variation of $\phi$ possesses an under- or overshoot; P denotes the centre of cell immediately upstream of the face $f$ in question; $\mathrm{U}$ is the second cell upstream and $\mathrm{D}$ is the downstream cell. Upstream and downstream directions are determined by the sign of the cell face flux $F_{\mathrm{f}}$. Fig. 2 shows two popular high-resolution schemes, the MINMOD of Harten [20], which combines the central differencing and secondorder upwind base schemes, and the SMART of Gaskell and Lau [21], which combines third-order upwind (often called QUICK, following Leonard [22]) with straight lines to pass through the points $(0,0)$ and $(1,1)$ in the NVD. Our new scheme CUBISTA is also shown, being represented by the following equations on a uniform mesh:

$$
\tilde{\phi}_{\mathrm{f}}= \begin{cases}1.75 \tilde{\phi}_{\mathrm{P}} & 0<\tilde{\phi}_{\mathrm{P}}<0.375, \\ 0.375+0.75 \tilde{\phi}_{\mathrm{P}} & 0.375 \leq \tilde{\phi}_{\mathrm{P}} \leq 0.75 \quad \text { (QUICK), } \\ 0.75+0.25 \tilde{\phi}_{\mathrm{P}} & 0.75<\tilde{\phi}_{\mathrm{P}}<1, \\ \tilde{\phi}_{\mathrm{P}} & \text { elsewhere } \quad \text { (UDS). }\end{cases}
$$

Although in the figure the representation of our scheme in the normalised variable diagram does not seem too different from SMART, the differences are sufficient to impart enhanced stability whenever CUBISTA is employed in conjunction with fully-implicit solution methods.

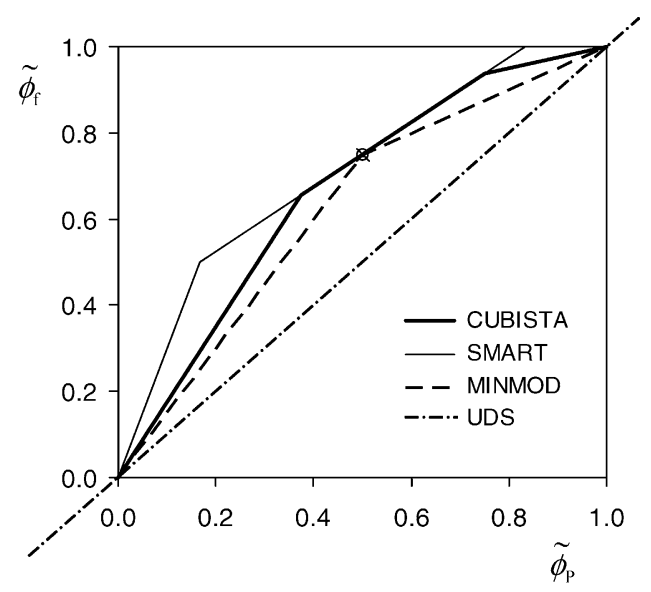

Fig. 2. Some high-resolution schemes in the normalised variable diagram. 
The basic ideas behind CUBISTA are given in the original paper [1]. Basically, it is composed of a portion of QUICK, therefore retaining the third-order accuracy in the smooth flow region (approximately $0.4 \leq \tilde{\phi}_{\mathrm{P}} \leq 0.6$, where gradients are mild), but then the two straight lines connecting with the origin and the unit point in the NVD diagram were designed to yield a symmetric scheme and to respect TVD restrictions. It is compliance with these restrictions that ensures the better iterative-convergence properties of this scheme compared with others, which often suffer from "switching" or other types of numerical-related instabilities that hinder convergence to a steady state solution. Some of the good qualities of the CUBISTA scheme in terms of robustness and accuracy, as compared with the upwind scheme for example, are illustrated by the next few examples.

The performance of the CUBISTA scheme is now illustrated in Figs. 3 and 4, for a flow related to the main problem of this work: the $4: 1$ contraction, with the Oldroyd-B model $(R e=0 ; \beta=1 / 9)$. Accuracy is qualitatively demonstrated in Fig. 3, where streamlines obtained on a medium mesh (number of cells $=5282$ ) are shown for a Deborah number of $D e$ $=2.5$, with the calculations based on the upwind differencing scheme (UDS) and the three high-resolution schemes of Fig. 2 (MINMOD, SMART and CUBISTA). For reference, a "mesh-converged" solution obtained with the CUBISTA scheme on a very fine mesh (number of cells $=169392$ ) is also shown. The upwind scheme generates a large vortex
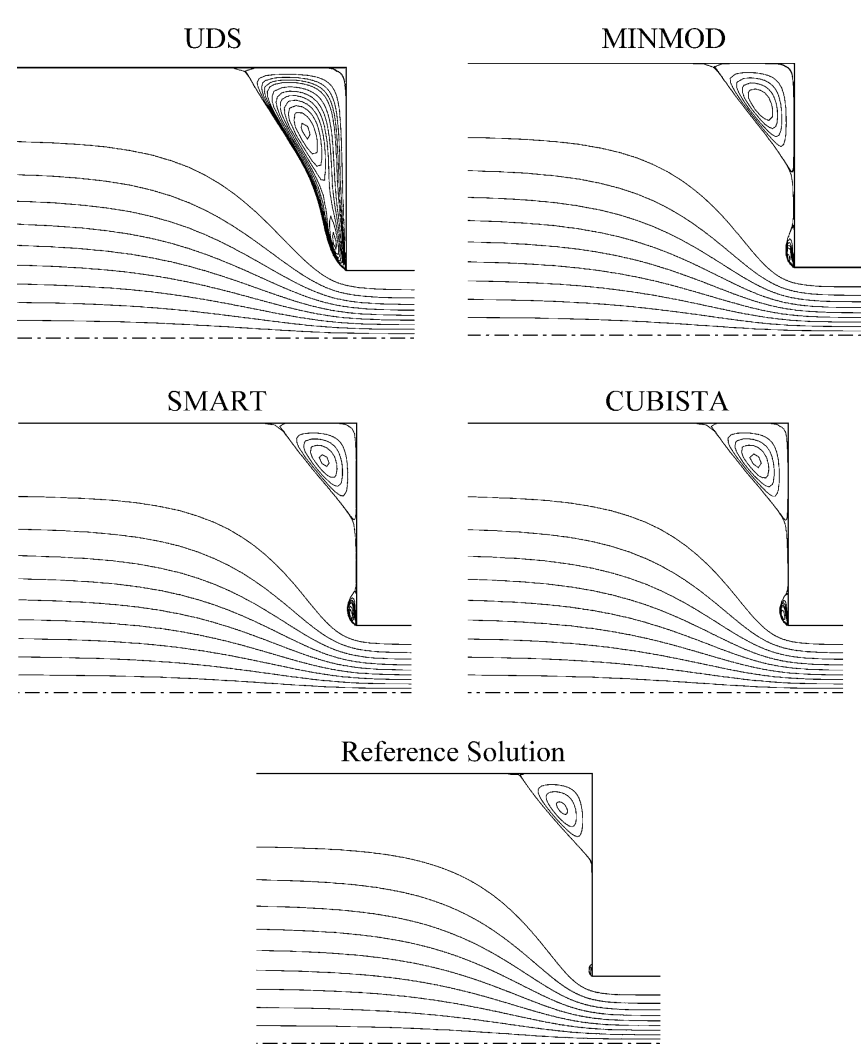

Fig. 3. Effect of new high-resolution scheme: accuracy. Streamlines for Oldroyd-B in 4:1 contraction at $D e=2.5$.
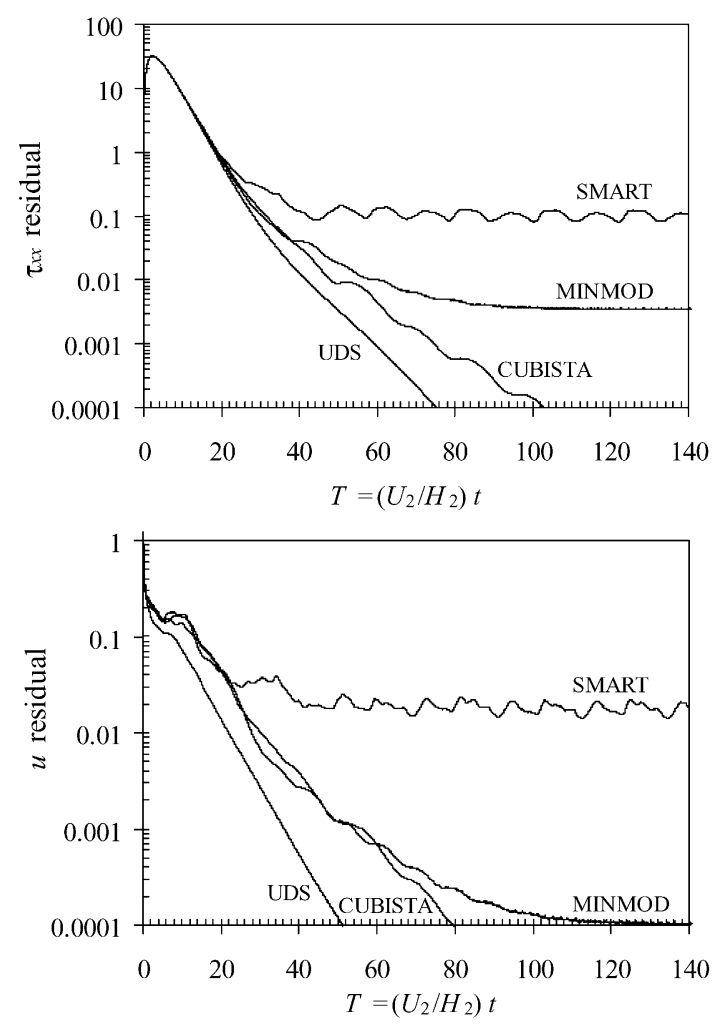

Fig. 4. Effect of new high-resolution scheme: robustness. Decay of residuals for Oldroyd-B in 4:1 contraction at $D e=3$.

which bears little resemblance with the "true" solution on the very fine mesh. Results with SMART and CUBISTA are almost undistinguishable and, on that very affordable mesh, are qualitatively very close to the "reference" results based on the much more expensive calculation with the fine mesh. Other more quantitative demonstrations of accuracy regarding rootmean-square errors and solution functionals are given in the original article [1].

Robustness, in terms of enhanced stability to yield an iterative-converged solution, is demonstrated in Fig. 4, where the time-evolution of the axial stress and velocity residuals are shown for the case $D e=3.0$. Both the MINMOD and SMART schemes are unable to meet the iterative-convergence criterion of $10^{-4}$ : the residuals stagnate at some level above this value, meaning that at some points on the mesh the equations are not being solved correctly. The CUBISTA, on the other hand, converges well to the steady state solution, as shown by the gradual decay of the residuals of the governing equations.

\section{Problem definition and computational meshes}

The configuration of the flow is illustrated in Fig. 1. The contraction ratio is here the main independent parameter, being defined as:

$\mathrm{CR}=\frac{H_{1}}{H_{2}}$, 
where $H_{1}$ is the half-height of the larger, incoming channel and $\mathrm{H}_{2}$ is the half-height of the smaller, outflow channel. Other dimensionless groups characterising this flow are the solvent viscosity ratio defined in Section $3, \beta \equiv \eta_{\mathrm{s}} / \eta_{0}=1 / 9$, the extensional parameter of the PTT model, here taken as $\varepsilon=0.25$ typical of polymer melts or concentrated solutions, and the Deborah number defined by:

$D e=\frac{\lambda U_{2}}{H_{2}}$,

where $U_{2}$ is the cross-sectional average velocity in the smaller channel. Inertia would be measured by a Reynolds number but, for the creeping flow conditions considered in this investigation, this parameter plays no role $(R e=0)$. The shear and extensional rheometrical properties for our PTT model were presented in Fig. 23 of Ref. [7], which is not repeated here, but inspection of that figure may be useful to interpret the results of the current simulations.

Some information about the computational meshes is given in Table 1 which includes the lengths of the upstream and downstream channels $\left(L_{1}\right.$ and $\left.L_{2}\right)$, the total number of cells inside the computational domain (NC), the number of degrees of freedom (DOF), and the size of the smallest cell. These meshes are non-uniform with cell clustering around the problematic re-entrant corner, and also along the channel walls due to the velocity and stress boundary layers that may develop there. It is quite clear that the channel lengths $L_{1}$ and $L_{2}$ need to be varied according to the contraction ratio (and also $D e$ ) so that adequate dimensions are provided for flow development and redevelopment upstream and downstream of the contraction plane. Guidelines for the choice of appropriate channel lengths as a function of $D e$ were given in a previous work ([7], for $\mathrm{CR}=4$ ) on the basis of the distances required for the normal stress $\tau_{x x}$ to be within $1 \%$ of its fully developed value; extension to higher $\mathrm{CR}$ is straightforward and leads to the values given in Table 1. If $H_{2}$ is considered to be fixed at a unit value (in our "standard" scaling, $H_{2}$ is the characteristic length scale) then it is $H_{1}$ which is increased as CR increases. Illustrative example of meshes, for $\mathrm{CR}=10$ and 100, are given in Fig. 5, where only a zoomed region near the contraction plane is shown. What is important to stress at this point is that the mesh dimensions around the re-entrant corner are the same for all meshes (with the same minimum cell spacing of $\Delta x / H_{2}=0.02$ ) so that the local variations of the re-

Table 1

Some characteristics of the computational meshes

\begin{tabular}{rlllrl}
\hline $\mathrm{CR}$ & $L_{1} / H_{2}$ & $L_{2} / H_{2}$ & $\mathrm{NC}$ & \multicolumn{1}{c}{ DOF } & $\Delta x_{\min }=\Delta y_{\min }$ \\
\hline 10 & 2500 & 2500 & 10420 & 62520 & 0.020 \\
20 & 2500 & 2500 & 11956 & 71736 & 0.020 \\
40 & 2500 & 2500 & 15796 & 94776 & 0.020 \\
100 & 5000 & 5000 & 23920 & 143520 & 0.020
\end{tabular}

$\overline{\mathrm{CR}}$, contraction ratio; $L_{1}$, length of upstream channel; $L_{2}$, length of downstream channel; NC, number of cells; DOF, degrees of freedom; $\Delta x, \Delta y$, cell spacing.
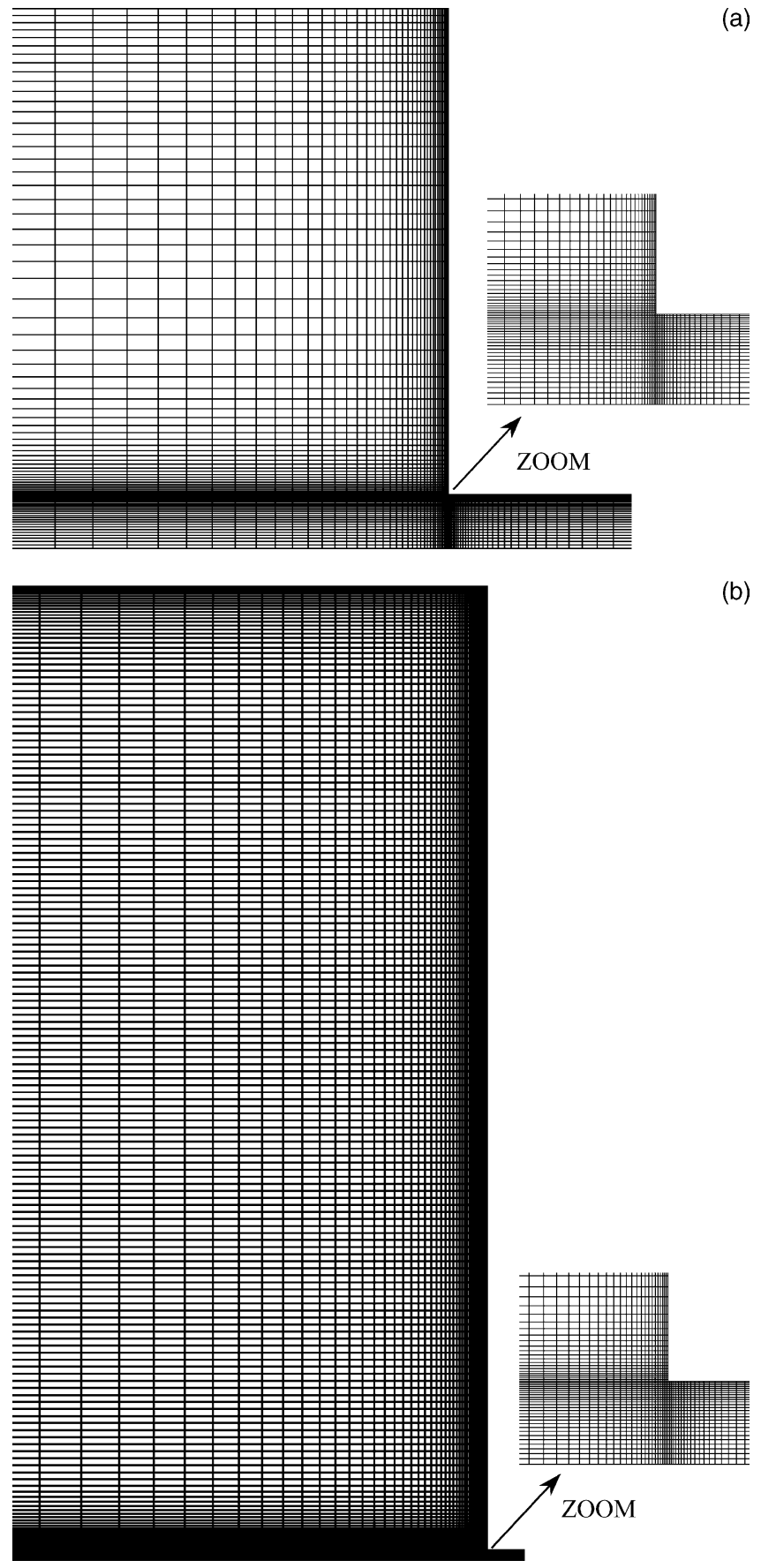

Fig. 5. (a) Zoomed view of the computational mesh for the contraction ratio 10:1. (b) Zoomed view of the computational mesh for the contraction ratio 100:1.

sulting solution fields are not influenced by the contraction ratio.

The imposed boundary conditions are:

- Analytical solutions for the velocity and stress variation at inlet $\left(x=-L_{1}\right)$; these were guided by the results of Oliveira and Pinho [23].

- No-slip conditions at the solid walls, with the stresses obtained from analytical plane Couette-flow solutions (at $y=$ 
$H_{1}$, for $x<0 ; y=H_{2}$, for $x>0$; and $H_{2} \leq y \leq H_{1}$, for $x=0$ ). We note that these boundary conditions for the stresses are required because the governing equations are not solved at the grid points on the wall.

- Vanishing streamwise gradients $(\partial / \partial x=0)$ at the downstream channel outlet $\left(x=+L_{2}\right)$.

- Symmetry conditions along the channels centreline $(y=$ 0 ), noting that the governing equations were solved in only half of the complete flow domain (cf. Fig. 1).

\section{Results}

We first give the predicted streamlines for the various contraction ratios (Section 6.1), to illustrate the existence of corner and lip vortices and, for a specific contraction ratio (CR =16) we do additional simulations to allow a more detailed comparison with the visualizations of Evans and Walters [6]. Then, we discuss the results for vortex size (Section 6.2), corner and lip vortex intensities (Section 6.3) and representation of vortex characteristics in a vortex map (Section 6.4). Finally, the proposed scaling rules are corroborated by means of comparison of local quantities (Section 6.5).

\subsection{Flow patterns}

It is well known [5,6] that, under creeping flow conditions, various types of vortices are formed upstream of the contraction plane. These vortices tend to increase with elasticity, for shear-thinning liquids, but may be of a different nature depending on the contraction ratio. A picture of some of the flow patterns predicted during the current work, for increasing Deborah numbers, is shown in Fig. 6 for $\mathrm{CR}=4$, in Fig. 7 for $\mathrm{CR}=20$, and in Fig. 8 for $\mathrm{CR}=100$. The results for $\mathrm{CR}$ $=4$ correspond to those already given in our previous benchmark work [7]. Since it will be shown in the next sections that the controlling parameter for corner vortex characteristics is not the standard Deborah number, but rather $D e / C R$, values of this ratio are also registered in the above figures.

In qualitative terms, Figs. 6-8 illustrate the vortex enhancement mechanism and highlight differences brought about by influence of the contraction ratio. For $\mathrm{CR}=4$, no lip vortex occurs for the PTT model with a linear stress function and $\varepsilon=0.25$. However, for higher CR, vortex enhancement seems always to be brought about by a lip vortex mechanism. Once a lip vortex is formed the tendency is for it to be intensified by elasticity (when $D e$ is raised), eventually engulfing the corner vortex and becoming the dominant near-contraction flow feature. This is pretty well illustrated by Fig. 7, for the 20: 1 contraction: at $D e=5$ a lip vortex is already present; such vortex is seen to dominate the secondary cell structure when $D e$ varies from 20 to 50; and then, for higher $D e$, a single remaining vortex exists, which is clearly related to the lip vortex (notice how the vortex centre is still located close to the entrance to the smaller channel). A closer inspection of these figures allows us to conclude that the condition $D e / C R$
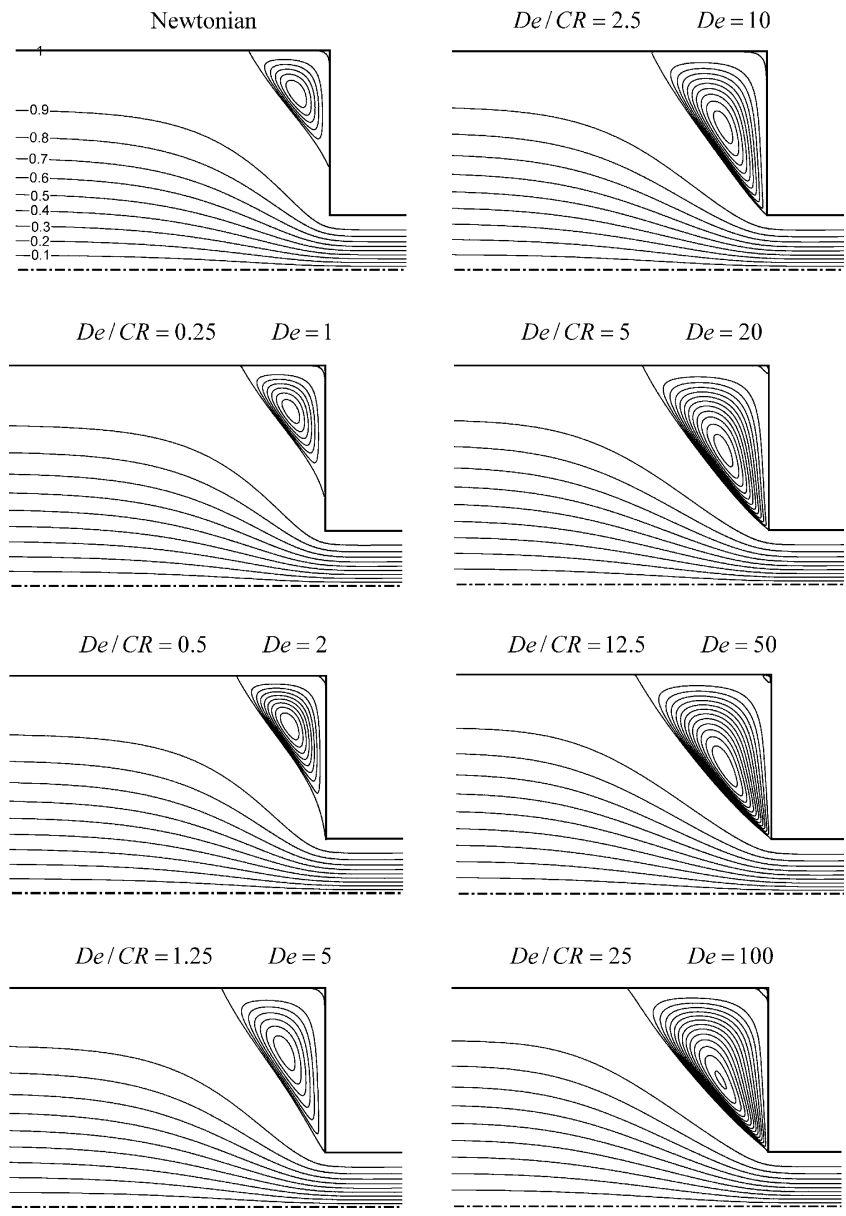

Fig. 6. Streamline patterns for the 4:1 contraction ratio.

$\approx 0.5-1$, when the two types of vortices are still simultaneously present but the lip vortex starts becoming dominant, separates the phases of incipient and strong vortex enhancement. This conclusion seems to be valid for all contraction ratios but, of course, for $\mathrm{CR}=4$ there is no visible lip vortex; the point $D e / C R \approx 1$ corresponds then to the situation when the fingering of the salient corner vortex towards the re-entrant corner ends, and vortex enhancement starts. A visible effects is that the shape of the vortex near the re-entrant corner goes from concave to convex.

It is interesting to contrast Figs. 6-8 with the visualizations of Evans and Walters [6] for the $1 \%$ aqueous solution of polyacrylamide, given in their Figs. 8-10 for, respectively, $\mathrm{CR}=4,16$ and 80. As already commented in Section 2, the qualitative resemblance of predicted and visualized flow patterns is striking, and many of the main features described by Evans and Walters are replicated by the simulations:

- the lack of a visible lip vortex in the 4:1 contraction;

- the lip-vortex enhancement mechanism present in the higher CR cases;

- the coexistence of corner and lip vortices for a certain range of $D e$; 

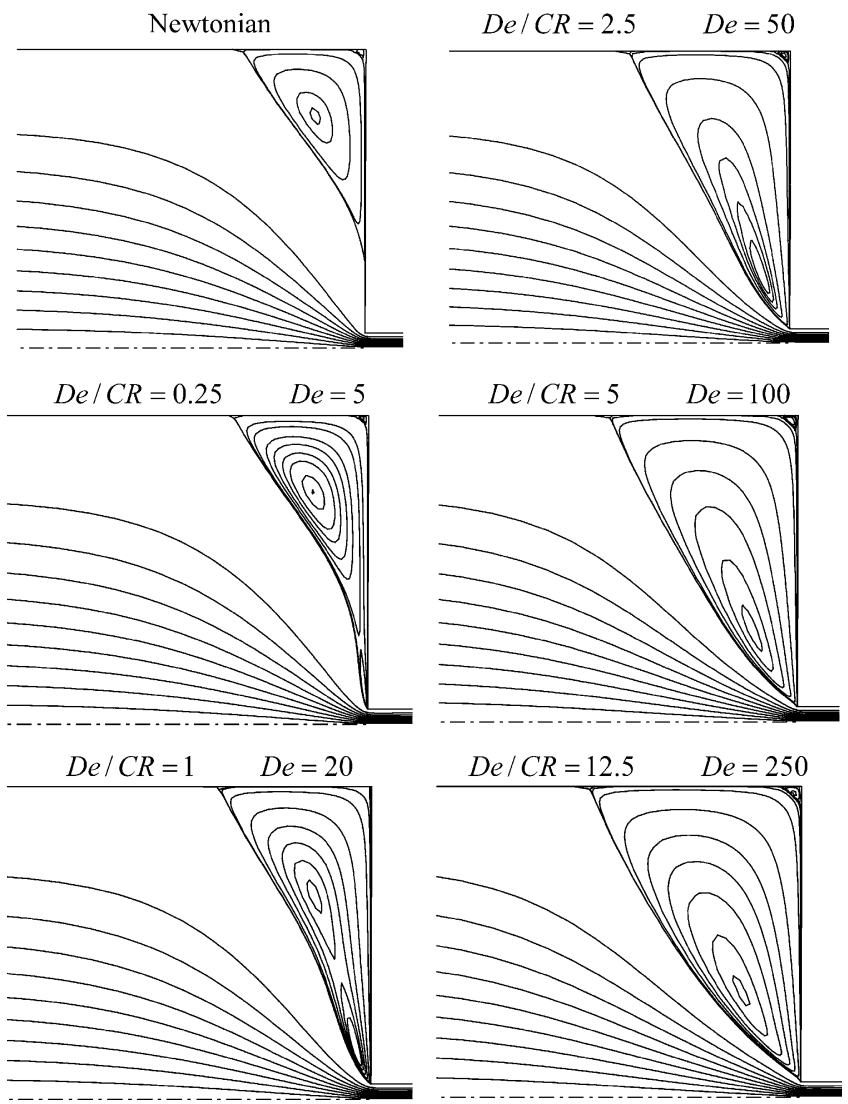

Fig. 7. Streamline patterns for the 20:1 contraction ratio.
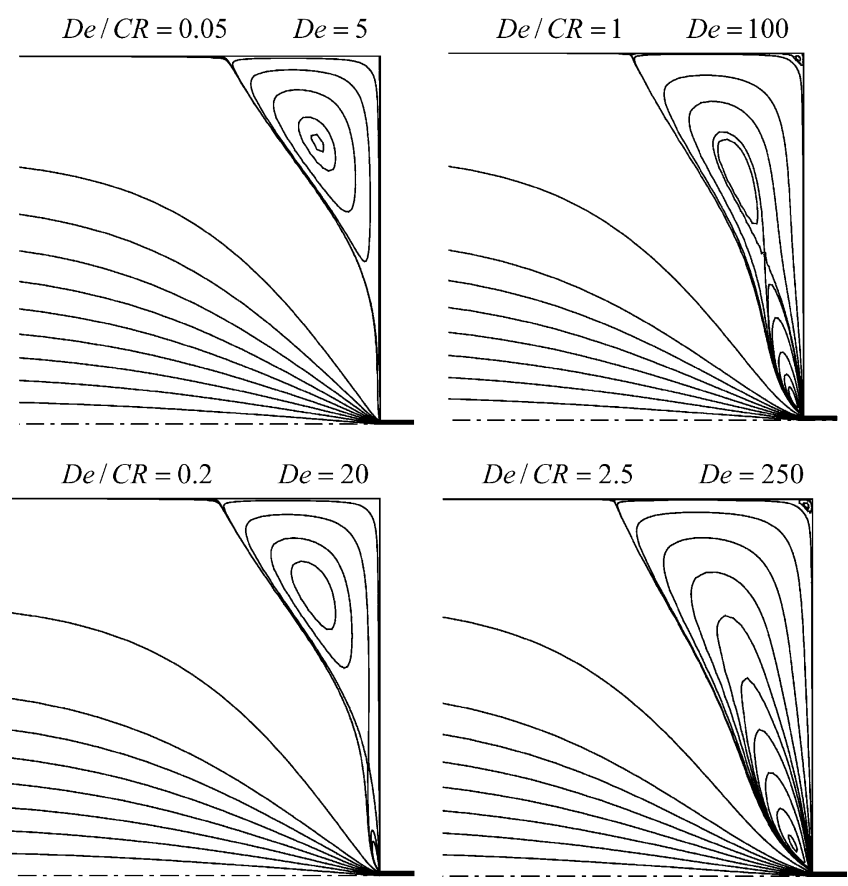

Fig. 8. Streamline patterns for the 100:1 contraction ratio.
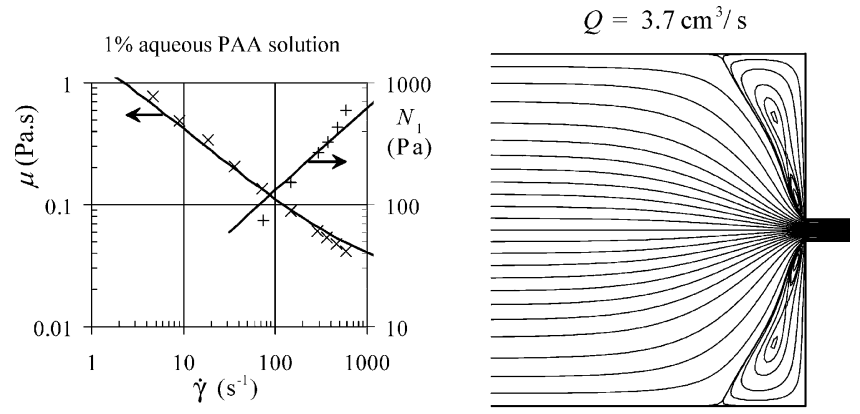

$Q=0.6 \mathrm{~cm}^{3} / \mathrm{s}$

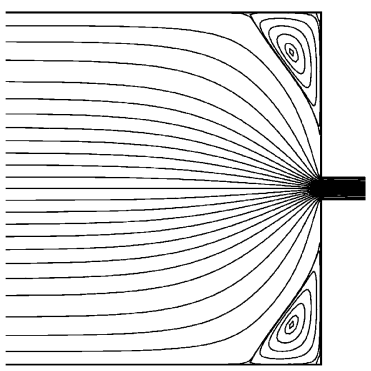

$Q=4.63 \mathrm{~cm}^{3} / \mathrm{s}$

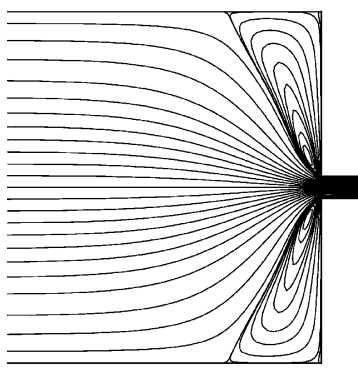

$Q=2.63 \mathrm{~cm}^{3} / \mathrm{s}$

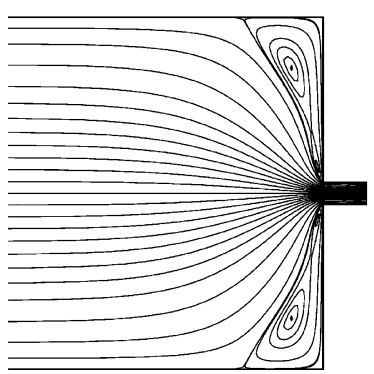

$Q=5.9 \mathrm{~cm}^{3} / \mathrm{s}$

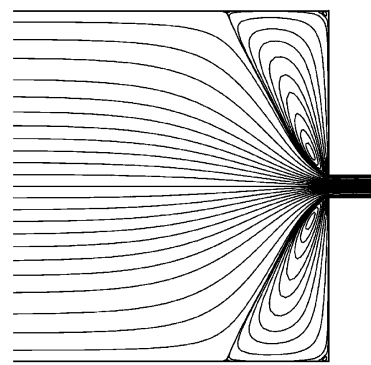

Fig. 9. Predictions of the flow considered by Evans and Walters [6] in a 16:1 planar contraction. The Deborah number varies from $D e=1.2(Q=$ $\left.0.6 \mathrm{~cm}^{3} / \mathrm{s}\right)$ to $D e=11.8\left(Q=5.9 \mathrm{~cm}^{3} / \mathrm{s}\right)$ and inertia is not important $(R e<$ $0.015)$.

- the strong lip vortices, with the vortex centres located close to the smaller-channel entrance, characteristic of the highest $\mathrm{CR}$ case.

Since the main interest of the current work was, at the outset, purely numerical, no effort was made to represent the rheology of any particular liquid. The PTT equation and the specific parameters $\varepsilon=0.25$ and $\beta=1 / 9$ were chosen so that the present results could be compared with previous numerical work [7]. However, the qualitative agreement with the experiments of Evans and Walters calls for a more (semi) quantitative comparison. The viscometric data given by those authors for the $1 \%$ aqueous solution of PAA can be approximately represented by the PTT model with the parameters $\lambda$ $=2 \mathrm{~s}, \varepsilon=0.25, \eta_{0}=2.5 \mathrm{~Pa}$ and $\beta=1 / 120$, as shown on the top left part of Fig. 9. A tighter fitting of the shear viscosity and first normal stress difference would require a multimode version of the PTT model, a matter left for future work. In Fig. 9 the results of the simulations are shown, as streamline patterns, for exactly the same dimensional conditions of the experiments in the 16:1 contraction, where the volumetric 
flow rates $Q$ were gradually increased (see Fig. 9 in [6]). We consider the agreement between numerics and experiments to be now, at least, of a semi-quantitative nature: absence of lip vortex at the lowest flow rate $Q=0.6 \mathrm{~cm}^{3} / \mathrm{s}$; formation of a small lip vortex at $Q=2.63 \mathrm{~cm}^{3} / \mathrm{s}$; co-existence of corner and lip vortices at $Q=3.7 \mathrm{~cm}^{3} / \mathrm{s}$ (see the zoomed photograph in the original Evans and Walters' work-Fig. 9D in [6]); and complete engulfment of the corner by the lip vortex by $Q=$ $4.63 \mathrm{~cm}^{3} / \mathrm{s}$.

It is satisfying to see that the PTT model with $\varepsilon=0.25$ does a very good job in predicting accurately the complex flow structures observed when a highly elastic polymer solution (1\% PAA) flows through a planar contraction. In particular, the effects of contraction ratio on flow patterns are well captured by the simulations with the PTT model. A close inspection of the photographs in [6] reveals some 3D effects (pathlines in the recirculation zones are not closed), which need to be taken into account in future numerical work.

\subsection{Prediction of corner vortex size}

The variation of the predicted corner vortex size with the Deborah number is shown in Fig. 10. Here, the standard scaling is employed, that is lengths are normalised with downstream channel half-height $H_{2}$ (we denote $X_{\mathrm{R}} \equiv x_{\mathrm{R}} / H_{2}$, where $x_{\mathrm{R}}$ is the longitudinal size of the corner vortex). It is seen that different curves exist for the various contraction ratios and, in each case, the vortex size increases with $D e$ and a tendency is observed, with the log-log scale utilised, for a slowdown of that rate of increase at high $D e$. It is well known that $H_{1}$ is the proper characteristic length for scaling when the contraction ratio is varied $\left(2 \mathrm{H}_{1}\right.$ is often used; or, in the corresponding axisymmetric problem, the diameter of the upstream tube). This is evident in Newtonian flow calculations and experimental observations and is also common in non-Newtonian inelastic and elastic problems (see Boger [5]). Guided by these facts, in Fig. 11 we plot the variation of $X_{\mathrm{R}} / \mathrm{CR}=x_{\mathrm{R}} / H_{1}$ with $D e$ (the raw data are the same as in Fig. 10) and although the figure shows a better correlation, compared with Fig. 10, there is still no universal behaviour. In fact, Fig. 11 is as good

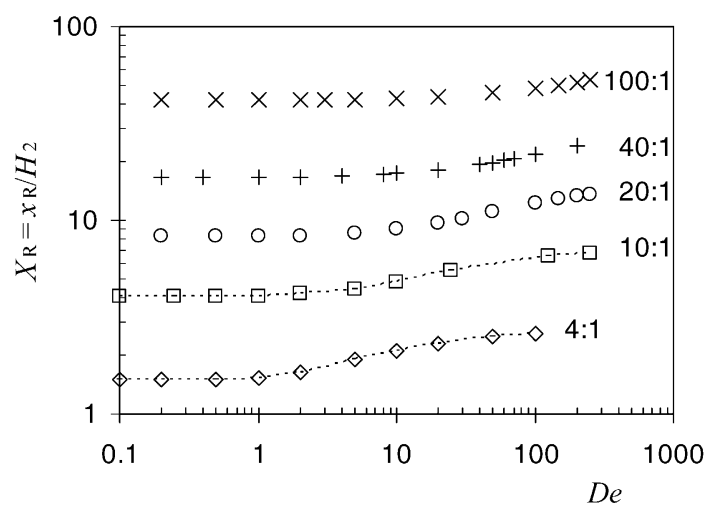

Fig. 10. Variation of corner vortex size with Deborah number: standard scaling.

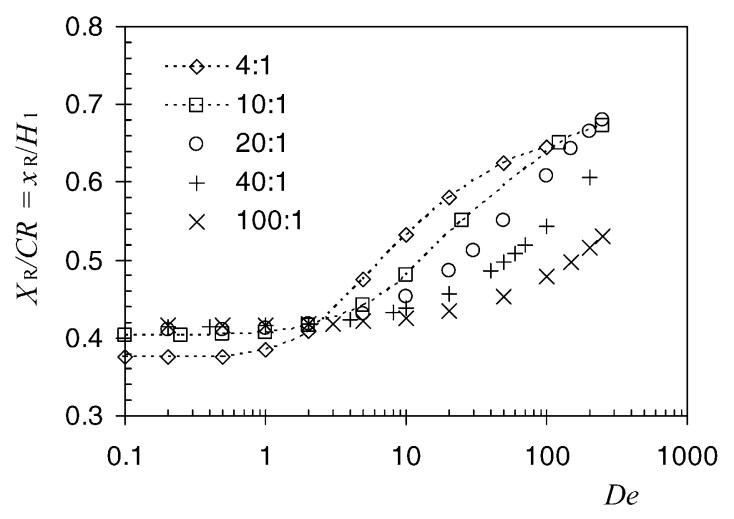

Fig. 11. Variation of corner vortex size, scaled with upstream channel halfheight, with Deborah number.

as the data scatter seen in Fig. 6 of Nguyen and Boger [4] where experimental measurements of $x_{\mathrm{R}} / D_{1}$, for various CR and fluids, were represented as a function of a Weissenberg number (the relation of this to our $D e$ is an open question, but the guidelines of [24] should be followed). That same figure, with perhaps some additional data points, is also given in a number of other publications by Boger and co-workers (see e.g. [5,9]). In an effort to achieve a tighter correlation, Nguyen and Boger [4] proposed to represent $(\mathrm{CR} \times W e) /\left(x_{\mathrm{R}} / D_{1}\right)$ as a function of an elasticity number $E l \equiv \lambda \eta_{0} / \rho D_{2}$ (note that an axisymmetric contraction was used in that work), but their data are better correlated only in an intermediate region of $E l$.

Based on intuition, we decided to re-plot our numerical data as $X_{\mathrm{R}} / \mathrm{CR}$ versus $D e / \mathrm{CR}=\lambda U_{2} / H_{1}$, and this is shown in Fig. 12. Clearly, a very good collapse of data is observed for CR $>10$ (see Fig. 12b). For $4 \leq \mathrm{CR}<10$ such a good agreement was not expected because the flow features in the salient corner region are too much influenced by what is happening inside the smaller channel. However, for high CR, the characteristics of the corner vortex are only affected by upstream events. Other characteristic length scales were tried for the variation of $x_{\mathrm{R}} / \ell$ versus $\lambda U_{2} / \ell$, for example $\ell=H_{1}-H_{2}$ and $\ell=\sqrt{H_{1}\left(H_{1}-H_{2}\right)}$, but none gave as good a collapse of the various curves as the choice $\ell=H_{1}$ of Fig. 12. This figure also serves to reinforce the conclusion of Section 6.1, showing that for $\mathrm{De} / \mathrm{CR}>0.5$ (approximately) the vortex size increases abruptly.

\subsection{Prediction of vortex intensities}

Fig. 13 shows the variation of the salient corner vortex intensity with the suggested Deborah number scaling, i.e. $D e / C R$, for the various contraction ratios; in part (a) all data are plotted while in part (b) only data for CR $>10$ are shown. Again, a reasonable correlation is achieved when $\Psi_{\mathrm{R}}$ is plotted versus $D e / \mathrm{CR}$, as occurred above with the vortex size. The $\Psi_{\mathrm{R}}$ in this figure is a measure of the fluid entrapped in the secondary cell and is given by the recirculating flow rate in the corner vortex divided by the inlet flow rate into the contraction domain. From the results in Fig. 13 one 


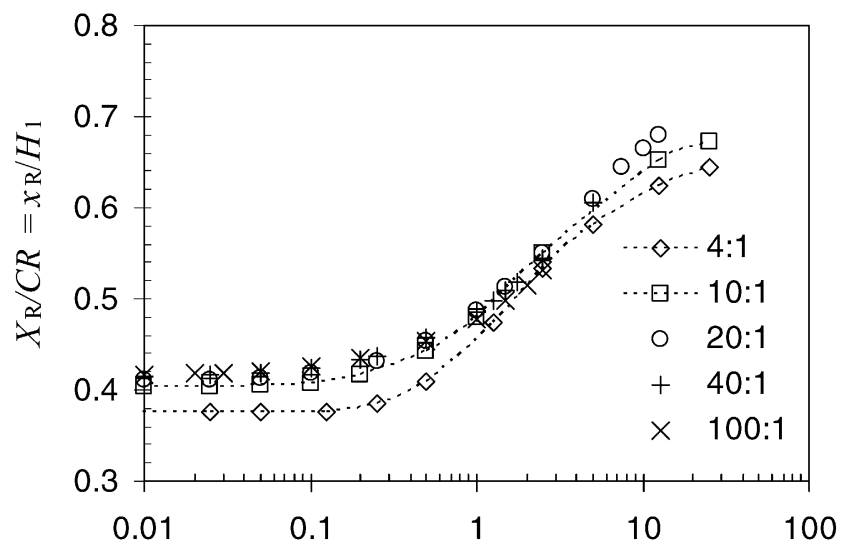

(a)

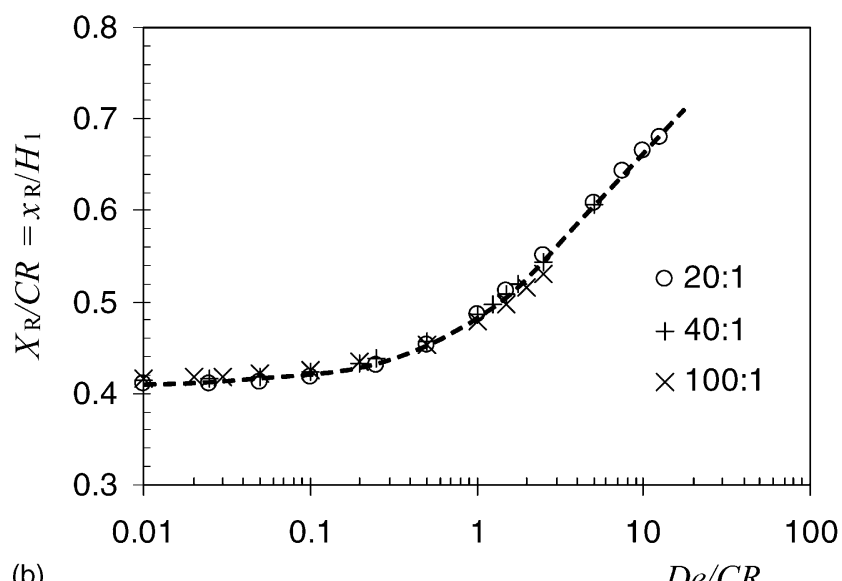

(b)

\section{$\mathrm{De} / \mathrm{CR}$}

Fig. 12. Variation of corner vortex size with modified scaling: (a) all contraction ratios; (b) contraction ratios greater than 10 .

may conclude that $D e / C R$ is the proper scaling parameter for the corner vortex intensity, which increases almost linearly with $D e / C R$ (the thick dashed line in Fig. $13 \mathrm{~b}$ is $\left.\Psi_{\mathrm{R}} \times 10^{3} \approx 1.65+2.4 \mathrm{De} / \mathrm{CR}\right)$.

However, when the intensity of the lip vortex, $\Psi_{\mathrm{L}}$, is considered an altogether different conclusion is reached, as demonstrated in Fig. 14 where $\Psi_{\mathrm{L}}$ is shown as a function of $D e$ alone, for various contraction ratios. It should be pointed out that the lip vortex intensity is here taken as representing the situations when a lip and a corner vortex exist simultaneously, or when the lip vortex merges with the corner vortex. It is quite clear from the preceding sections that the lip vortex, once formed, quickly grows with $D e$ to dominate the corner vortex and eventually completely engulfs that vortex to become the single remaining vortex structure.

In Fig. 14(a) the increase in intensity is shown in log-log scale, while Fig. 14(b) shows the same data on a linear scale. The rate of growth of lip vortex intensity is not linear with De. It appears from Fig. 14 that the lip vortex intensity tends to grow as a function of the Deborah number raised to the power $2 / 3$, as $D e$ gets higher. The fact that the lip vortex characteristics scale with $D e$ was also apparent from the experimental data of McKinley et al. [11] for vortex size (see
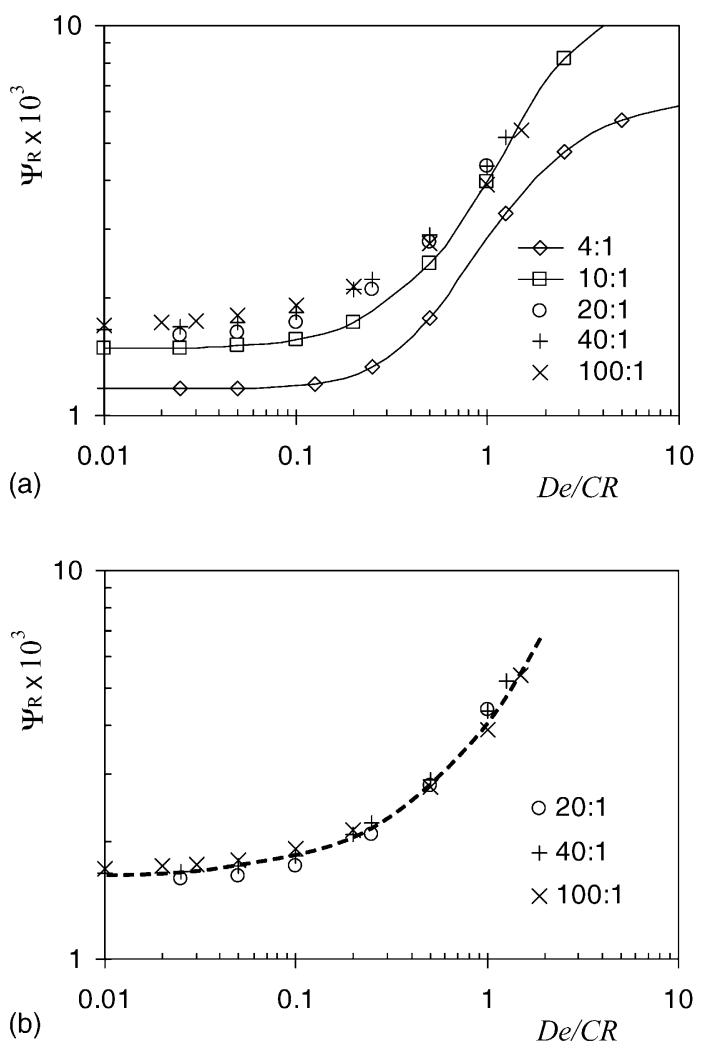

Fig. 13. Variation of corner vortex intensity with modified $D e$ scaling: (a) all contraction ratios; (b) contraction ratios greater than 10 .
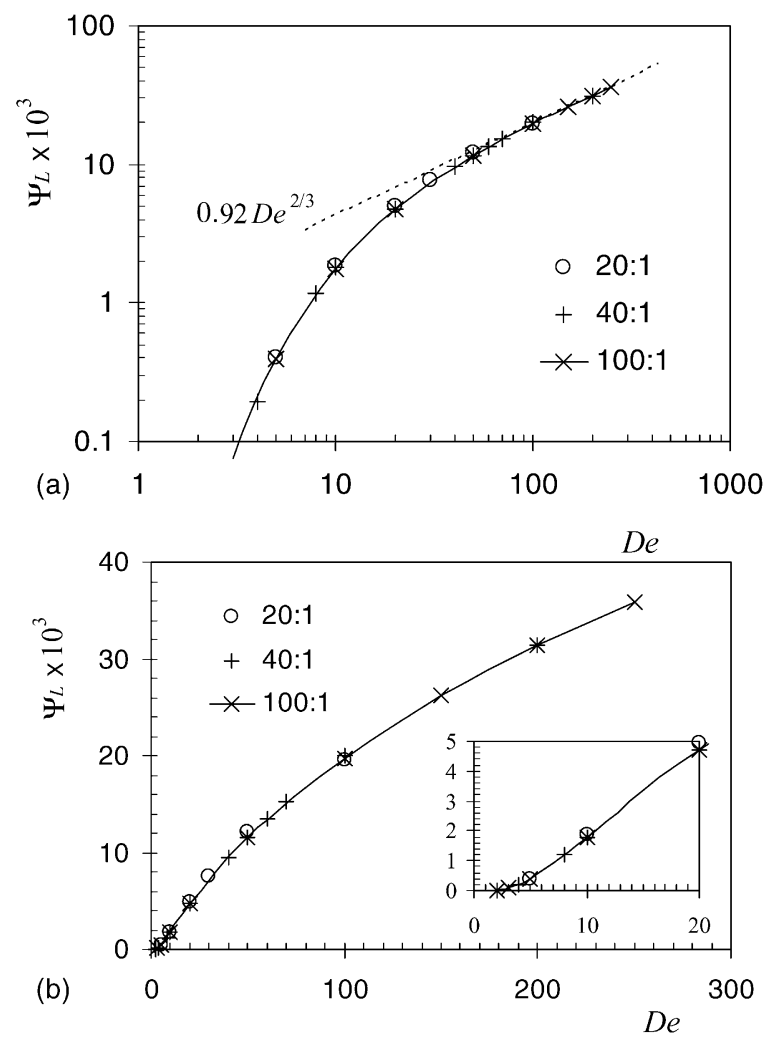

Fig. 14. Variation of lip vortex intensity with standard De scaling. 
their Fig. 4), although it is not straightforward to reconcile that with our conclusions because that data is for a Boger fluid in axisymmetric contractions at relatively low CRs.

\subsection{Map of vortex patterns}

With a view to classifying the structure of flows through contractions, we have carried out an extensive simulation programme, for various contraction ratios and increasing Deborah numbers, and the resulting steady-state flow patterns in the contraction plane region were classified as corner vortex, lip vortex, or as a combination of lip plus corner vortex structures. In Fig. 15 the outcome of this classification is plotted as a map of vortex type versus contraction ratio and Deborah number. Since we have already demonstrated that corner vortex characteristics are better scaled with $D e / C R$, while lip vortex characteristics scale with $D e$ alone, Fig. 15(a) shows the vortex pattern map as a function of $D e / C R$ while Fig. 15(b) shows it as a function of $D e$.

These two figures demonstrate once again that lip vortex formation is controlled by $D e$ (see the vertical dividing line in Fig. 15(b)), while the onset of a single merged vortex is controlled by $D e / C R$ (vertical line on the right side of
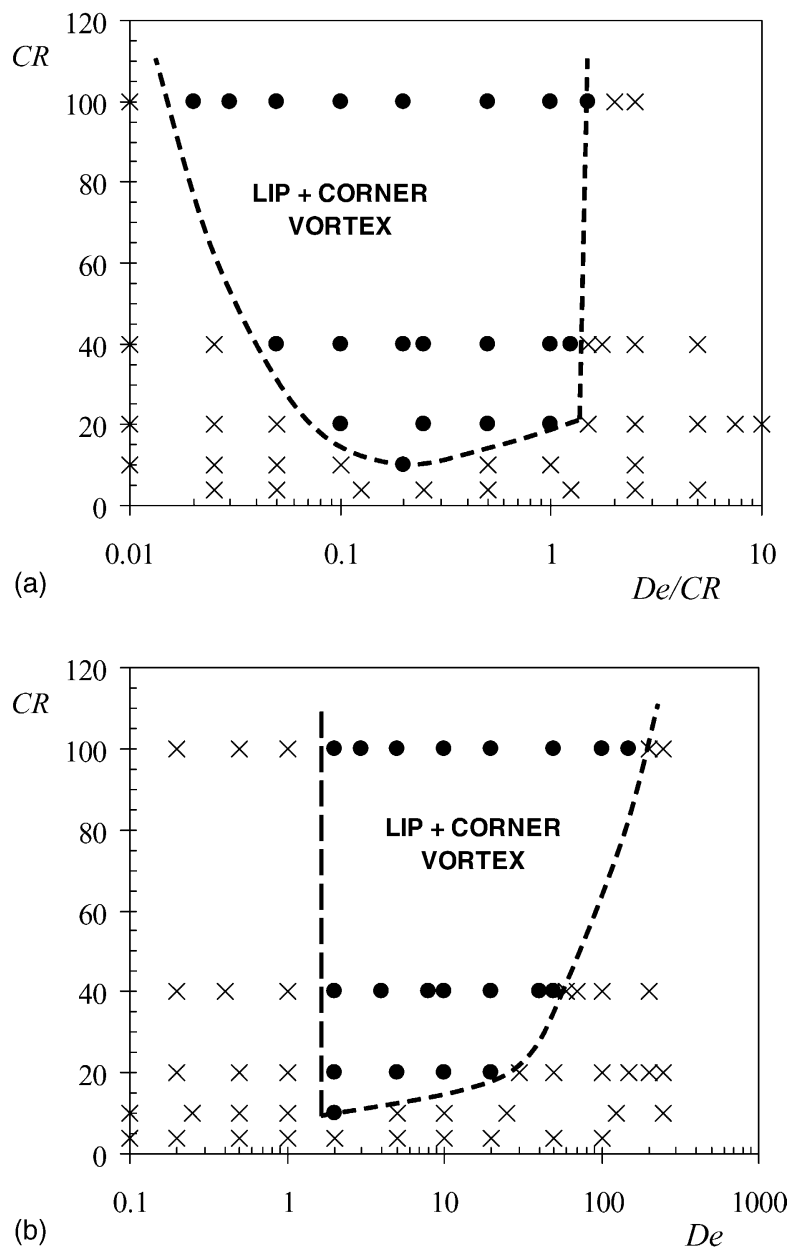

Fig. 15. Vortex pattern maps: (a) modified De scaling; (b) standard scaling.
Fig. 15(a)) even though its strength remains controlled by $D e$ (cf. Fig. 14). The sudden intensification of "vortex enhancement" correlates with the final stages of the co-existence of corner and lip vortices, when the latter starts becoming dominant, and it is seen from Fig. 15(a) to occur at around $D e / \mathrm{CR} \approx 1$. On the other hand, the formation of a lip vortex appears to occur at a fixed Deborah number for all contraction ratios except the lowest, and for the present constitutive model Fig. 15 (b) suggests a value of about $D e \approx 1-2$. For the lowest contraction ratio, $\mathrm{CR}=4$, Fig. 15 shows no sign of a lip vortex, in agreement with the experimental findings of [6]. Additional work is required to better fill the vortex maps of Fig. 15 for the PTT fluid, and especially to account for the effect of the model parameter $\varepsilon$, but the main features are well captured by the present results.

\subsection{Scaling of streamlines}

The evidence from the preceding sections provides already a clear indication of the correct scaling parameters for the overall characteristics of corner and lip vortices in viscoelastic contraction flows. Here some additional evidence is given regarding the scaling of local flow characteristics, as opposed to the global variables of Sections 6.2 and 6.3.

We deal first with corner vortex scaling. Fig. 16 shows the streamlines for two Deborah numbers $(D e=10$ and 50) in two different contraction geometries $(C R=20$ and 100).
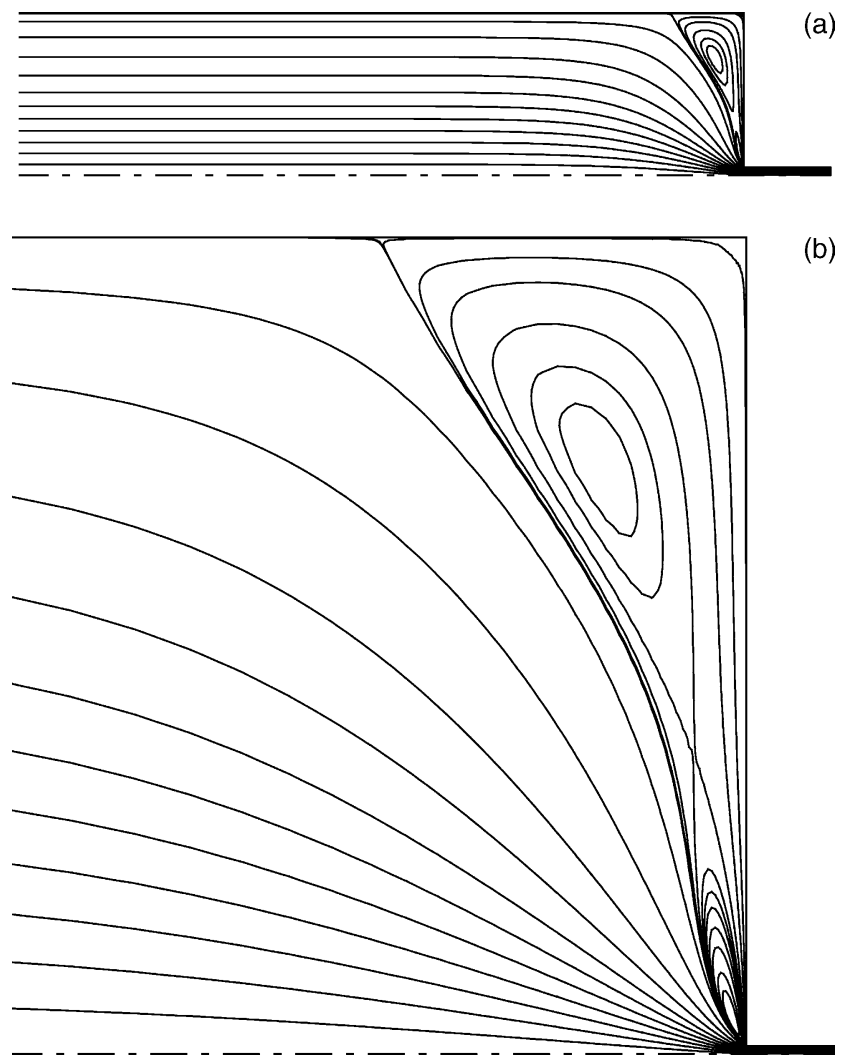

Fig. 16. Comparison of streamlines for two contraction ratios under standard scaling: (a) $\mathrm{CR}=20, D e=10$; (b) $\mathrm{CR}=100, D e=50$. 


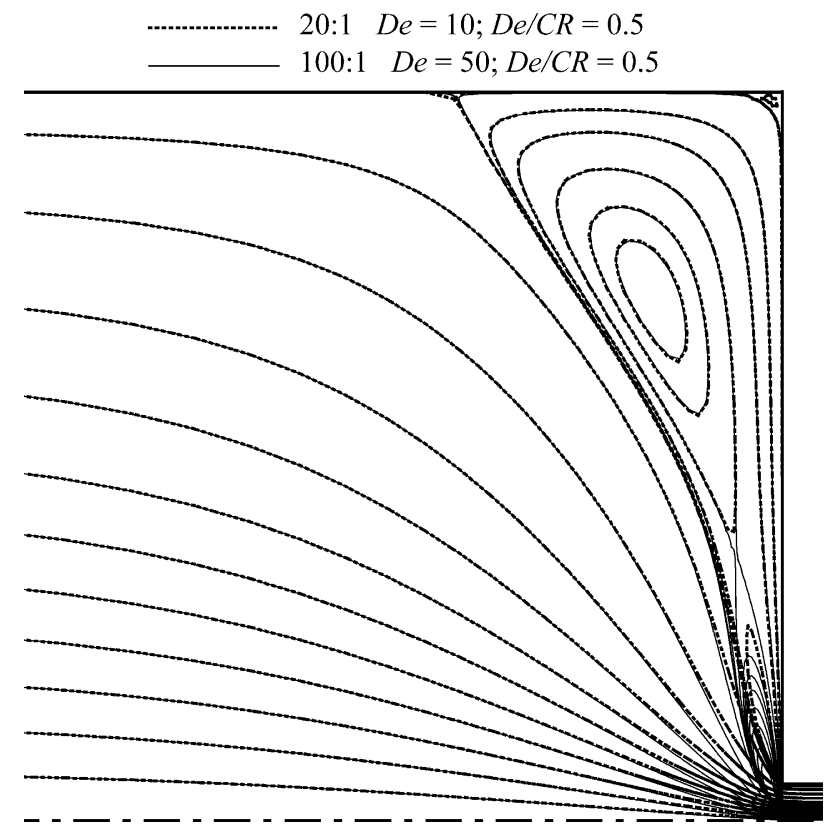

Fig. 17. Comparison of streamlines after scaling distances with upstream channel height (modified scaling).

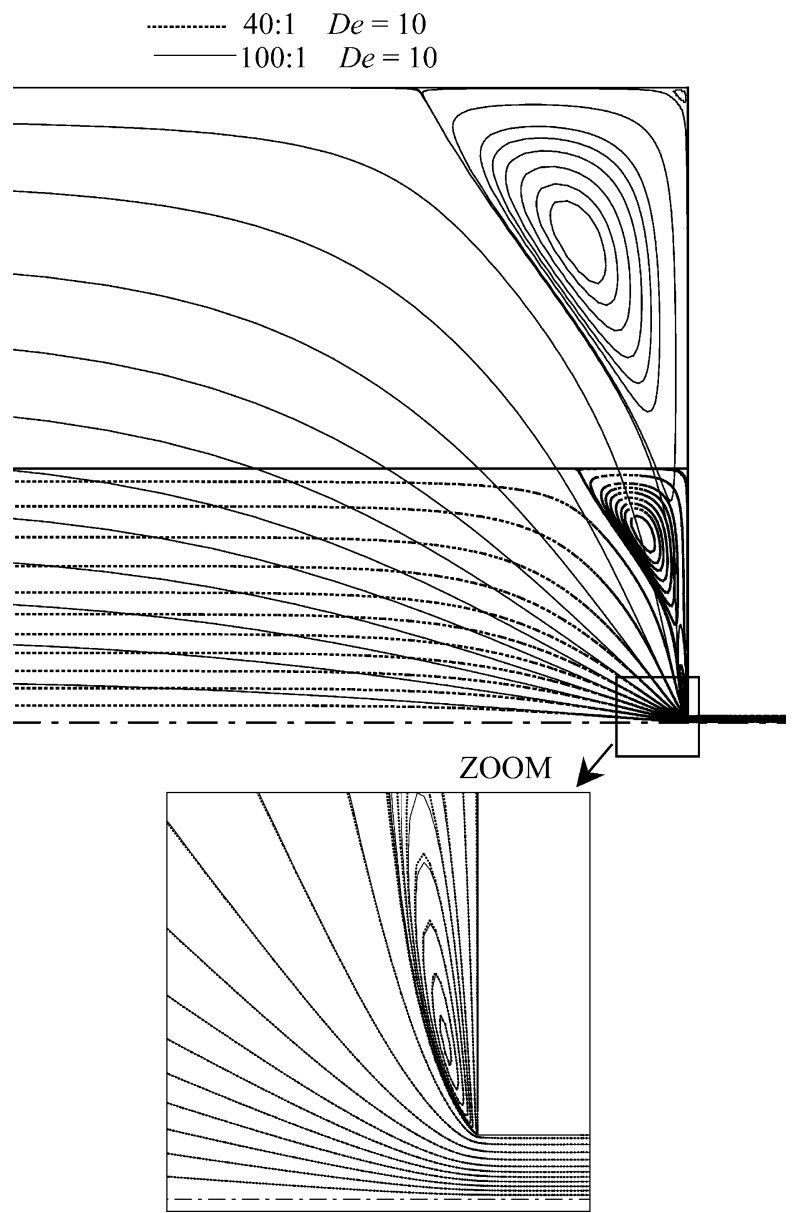

Fig. 18. Comparison of streamlines for two contraction ratios $(\mathrm{CR}=40$ and 100) under standard scaling $(D e=10)$.
However, the values of Deborah number divided by contraction ratio are identical, $D e / \mathrm{CR}=0.5$. Now, in order to check whether some similarity exists between these two flow patterns upon re-scaling, distances are normalised with $H_{1}$ (or $\mathrm{CR}$, in non-dimensional terms) and the resulting streamlines are superimposed, as shown in Fig. 17. Clearly, away from the entrance region a perfect match of streamlines is achieved; this means that the flow field inside the salient corner vortex scales with the dimensionless group $D e / C R$ when distances are normalised with upstream channel dimensions. This is a new result that we find very interesting and could certainly not have been anticipated.

Now we consider the lip vortex. Fig. 18 shows superimposed streamlines for two contraction ratios, 40:1 and 100:1, at the same $D e=10$ (in this case $D e / \mathrm{CR}$ is different, being 0.25 and 0.1 , respectively). A replot of these two flow fields, following the same procedure of Fig. 17, would show that the corner vortex characteristics do not scale under those conditions because the $D e / C R$ are different (for conciseness, such a figure is not given). However, by zooming into the

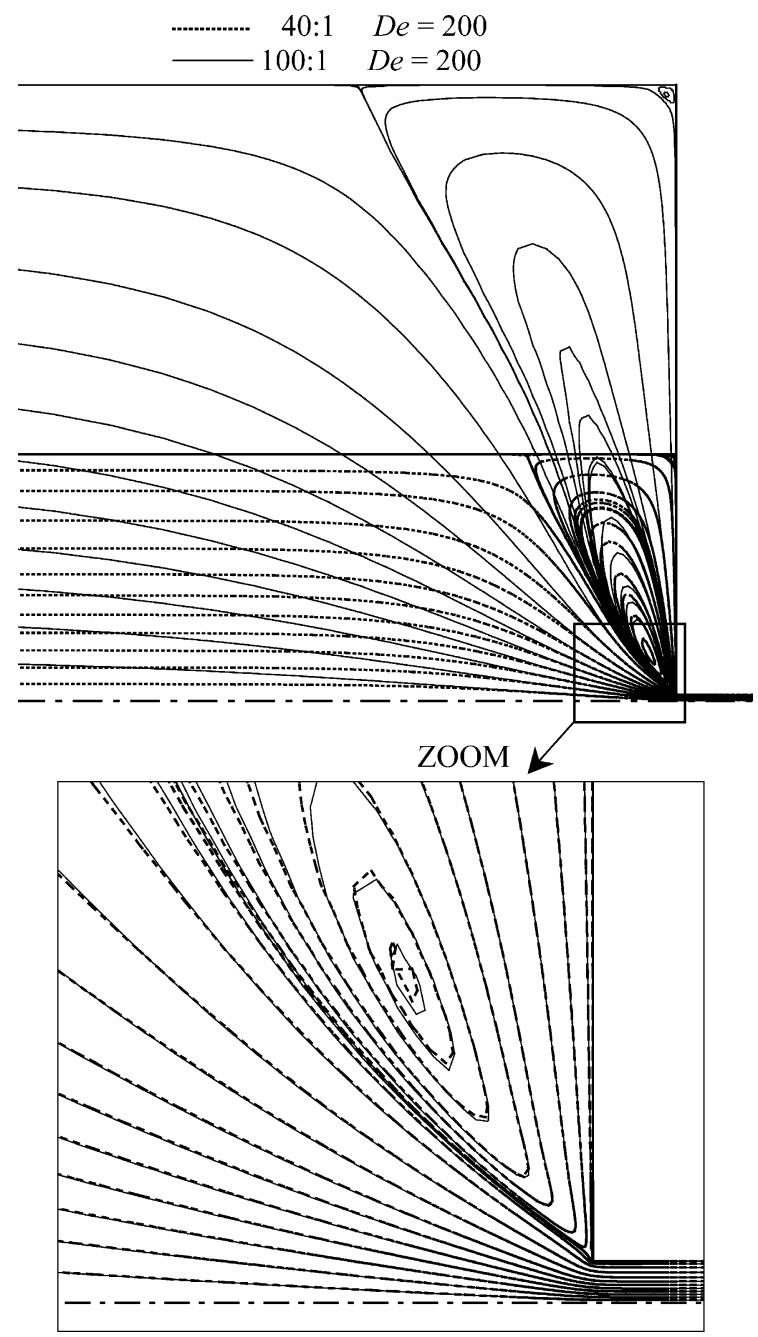

Fig. 19. Comparison of streamlines for two contraction ratios $(\mathrm{CR}=40$ and $100)$ under standard scaling $(D e=200)$. 
small lip vortex dimensions of Fig. 18, we see that lip vortex streamlines do coincide for the two conditions, thus demonstrating that lip vortices scale as $D e$. It may be argued that in Fig. 18 the lip vortex is still very small and is not yet the dominant flow structure. If we increase the elasticity of the fluid, by pushing $D e$ up to 200 (giving $D e / C R=5$ for $\mathrm{CR}=40$, and $D e / \mathrm{CR}=2$ for $\mathrm{CR}=100$ ), we observe a large lip-vortex-dominated structure as shown in Fig. 19. A zoom in the lip region for these two superimposed flow cases demonstrates once more that, for equal $D e$, streamline patterns do coincide. This result is perhaps not as interesting as the De/CR scaling of the corner vortex because, for the relatively large contraction ratios considered, the flow into the smaller channel approaches the conditions of an entry flow and should essentially be independent of upstream features.

\section{Conclusions}

Extensive numerical simulations of viscoelastic (PTT) liquid flow through planar contractions were carried out with a second-order finite volume method, for varying contraction ratios and Deborah numbers. The analysis of the results provided the following new findings and conclusions:

- For CR > 4, vortex enhancement follows a lip-vortex mechanism and the predicted streamlines show striking qualitative agreement with the experimental visualizations of Evans and Walters [6].

- When the shear material functions of the liquid used in [6] were more closely fitted to the PTT model, the agreement between the new simulations and the visualizations of [6] for the 16:1 contraction can be considered as semiquantitative. The range of flow rates in which there is coexistence of corner and lip vortex is well captured.

- From maps of vortex structure versus $C R$ and $D e$ or $D e / C R$, it can be drawn that the lip vortex is formed at $D e \approx 1-2$ for all contraction ratios (except $\mathrm{CR}=4$ ), and substantial vortex enhancement starts at $D e / \mathrm{CR} \approx 0.5-1$. For $\mathrm{De} / \mathrm{CR} \geq 1-2$ the corner vortex is completely engulfed by the lip vortex.

- Regarding universal correlations we have shown that global, as well as local, corner vortex flow features (size, intensity and streamlines) scale with upstream length scale ( $H_{1}$-half-height of larger channel) and with elasticity measured by $D e / \mathrm{CR}$ (where CR is the contraction ratio).

- Lip vortex intensity and streamlines scale with downstream length scale $\left(\mathrm{H}_{2}\right.$-half-height of smaller channel) and elasticity measured by $D e$.

- The above two conclusions are valid for relatively large contraction ratios, $\mathrm{CR}>10$. For lower contraction ratios $(4 \leq \mathrm{CR} \leq 10)$ both length scales $\left(H_{1}\right.$ and $\left.H_{2}\right)$ simultaneously influence the vortex features, as would be expected.

- It is worth emphasising that the above conclusions on scaling are valid both for global $\left(X_{\mathrm{R}}, \Psi_{\mathrm{R}}, \Psi_{\mathrm{L}}\right)$ and local (streamlines, an indirect measure of local velocities and stresses) vortex features.

In addition, the present results complement our previous work for the 4:1 contraction [7], by providing numerical data with sufficient accuracy to be used in assessment of computational rheology codes, for the contraction ratios 10, 20, 40 and 100, with the Phan-Thien/Tanner constitutive equation having a linear stress function and model parameters $\varepsilon$ $=0.25, \beta=1 / 9$, and $\xi=0$.

\section{Acknowledgements}

This work was in part funded by Fundação para a Ciência e Tecnologia (FCT, Portugal) and FEDER under project POCTI/EQU/37699/2001. M.A. Alves wishes to acknowledge the financial support provided by Fundação Calouste Gulbenkian. The authors would like to express their gratitude to the positive contributions from the referees.

\section{References}

[1] M.A. Alves, P.J. Oliveira, F.T. Pinho, A convergent and universally bounded interpolation scheme for the treatment of advection, Int. J. Numer. Meth. Fluids 41 (2003) 47-75.

[2] O. Hassager, in: Working group on numerical techniques, in: Proceedings of the Fifth Workshop on Numerical Methods in Non-Newtonian Flow, J. Non-Newton. Fluid Mech. 29 (1988) $2-5$.

[3] S. Nigen, K. Walters, Viscoelastic contraction flows: comparison of axisymmetric and planar configurations, J. Non-Newton. Fluid Mech. 102 (2002) 343-359.

[4] H. Nguyen, D.V. Boger, The kinematics and stability of die entry flows, J. Non-Newton. Fluid Mech. 5 (1979) 353-368.

[5] D.V. Boger, Viscoelastic Flows through contractions, Ann. Rev. Fluid Mech. 19 (1987) 157-182.

[6] R.E. Evans, K. Walters, Flow characteristics associated with abrupt changes in geometry in the case of highly elastic liquids, J. NonNewton. Fluid Mech. 20 (1986) 11-29.

[7] M.A. Alves, P.J. Oliveira, F.T. Pinho, Benchmark solutions for the flow of Oldroyd-B and PTT fluids in planar contractions, J. NonNewton. Fluid Mech. 110 (2003) 45-75.

[8] N. Phan-Thien, R.I. Tanner, A new constitutive equation derived from network theory, J. Non-Newton. Fluid Mech. 2 (1977) 353-365.

[9] D.V. Boger, D.U. Hur, R.J. Binnington, Further observations of elastic effects in tubular entry flows, J. Non-Newton. Fluid Mech. 20 (1986) 31-49.

[10] R.E. Evans, K. Walters, Further remarks on the lip-vortex mechanism of vortex enhancement in planar-contraction flows, J. Non-Newton. Fluid Mech. 32 (1989) 95-105.

[11] G.H. McKinley, W.P. Raiford, R.A. Brown, R.C. Armstrong, Nonlinear dynamics of viscoelastic flow in axisymmetric abrupt contractions, J. Fluid Mech. 223 (1991) 411-456.

[12] J.M. Maia, D. Binding, Influence of elongational properties on the contraction flow of polyisobutylene in a mixed solvent, Rheol. Acta 38 (1999) 160-171.

[13] M.A. Alves, F.T. Pinho, P.J. Oliveira, Effect of a high-resolution differencing scheme on finite-volume predictions of viscoelastic flows, J. Non-Newton. Fluid Mech. 93 (2000) 287-314.

[14] C. Béraudo, A. Fortin, T. Coupez, Y. Demay, B. Vergnes, J.F. Agassant, A finite element method for computing the flow of multi-mode 
viscoelastic fluids: comparison with experiments, J. Non-Newton. Fluid Mech. 75 (1998) 1-23.

[15] P.J. Coates, R.C. Armstrong, R.A. Brown, Calculation of steady-state viscoelastic flow through axisymmetric contractions with the EEME formulation, J. Non-Newton. Fluid Mech. 42 (1992) 141-188.

[16] M. Aboubacar, H. Matallah, M.F. Webster, Highly elastic solutions for Oldroyd-B and Phan-Thien/Tanner fluids with a finite volume/element method: planar contraction flows, J. Non-Newton. Fluid Mech. 103 (2002) 65-103.

[17] L.M. Quinzani, R.C. Armstrong, R.A. Brown, Use of coupled birefringence and LDV studies of flow through a planar contraction to test constitutive equations for concentrated polymer solutions, J. Rheol. 39 (1995) 1201-1228.

[18] P.J. Oliveira, F.T. Pinho, G.A. Pinto, Numerical simulation of nonlinear elastic flows with a general collocated finite-volume method, J. Non-Newton. Fluid Mech. 79 (1998) 1-43.

[19] B.P. Leonard, Bounded higher-order upwind multidimensional finitevolume convection-diffusion algorithms, in: W.J. Minkowycz, E.M.
Sparrow (Eds.), Advances in Numerical Heat Transfer, 1, Taylor and Francis, 1996, pp. 1-57.

[20] A. Harten, High-resolution schemes for hyperbolic conservation laws, J. Comput. Phys. 49 (1983) 357-393.

[21] P.H. Gaskell, A.K.C. Lau, Curvature compensated convective transport: SMART, a new boundedness preserving transport algorithm Int. J. Numer. Meth. Fluids 8 (1988) 617-641.

[22] B.P. Leonard, A stable and accurate convective modelling procedure based on quadratic interpolation, Comput. Meth. Appl. Mech. Eng. 19 (1979) 59-98.

[23] P.J. Oliveira, F.T. Pinho, Analytical solution for fully developed channel and pipe flow of Phan-Thien-Tanner fluids, J. Fluid Mech. 387 (1999) 271-280.

[24] D.V. Boger, M.J. Crochet, R.A. Keiller, On viscoelastic flows through abrupt contractions, J. Non-Newton. Fluid Mech. 44 (1992) 267-279. 